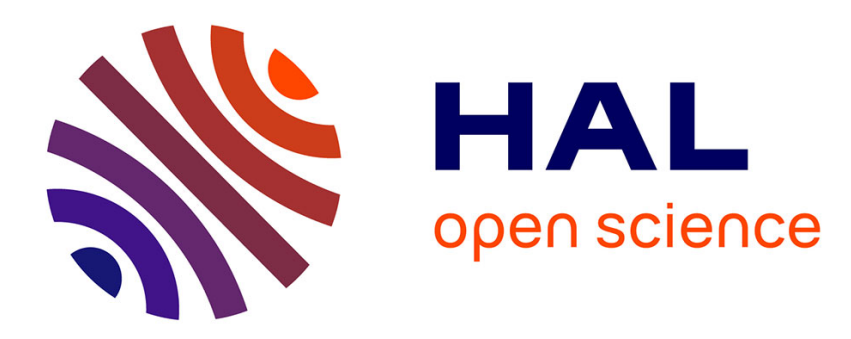

\title{
Analytic properties of the electromagnetic Green's function
}

Boris Gralak

\section{To cite this version:}

Boris Gralak. Analytic properties of the electromagnetic Green's function. Journal of Mathematical Physics, 2017, 58, pp.071501. 10.1063/1.4993199 . hal-01571649

\section{HAL Id: hal-01571649 \\ https://hal.science/hal-01571649}

Submitted on 13 Apr 2018

HAL is a multi-disciplinary open access archive for the deposit and dissemination of scientific research documents, whether they are published or not. The documents may come from teaching and research institutions in France or abroad, or from public or private research centers.
L'archive ouverte pluridisciplinaire HAL, est destinée au dépôt et à la diffusion de documents scientifiques de niveau recherche, publiés ou non, émanant des établissements d'enseignement et de recherche français ou étrangers, des laboratoires publics ou privés. 


\section{Analytic properties of the electromagnetic Green's function}

Boris Gralak

Citation: Journal of Mathematical Physics 58, 071501 (2017); doi: 10.1063/1.4993199

View online: http://dx.doi.org/10.1063/1.4993199

View Table of Contents: http://aip.scitation.org/toc/jmp/58/7

Published by the American Institute of Physics

\section{Articles you may be interested in}

Scattering for a 3D coupled nonlinear Schrödinger system

Journal of Mathematical Physics 58, 071502 (2017); 10.1063/1.4993224

Pedal coordinates, dark Kepler, and other force problems

Journal of Mathematical Physics 58, 063505 (2017); 10.1063/1.4984905

Superintegrable classical Zernike system

Journal of Mathematical Physics 58, 072901 (2017); 10.1063/1.4990793

Fock representations of $\mathrm{Q}$-deformed commutation relations

Journal of Mathematical Physics 58, 073501 (2017); 10.1063/1.4991671

The solution structure of the $\mathrm{O}(3)$ sigma model in a Maxwell-Chern-Simons theory Journal of Mathematical Physics 58, 071503 (2017); 10.1063/1.4994060

Hamilton-Jacobi approach to potential functions in information geometry Journal of Mathematical Physics 58, 063506 (2017); 10.1063/1.4984941

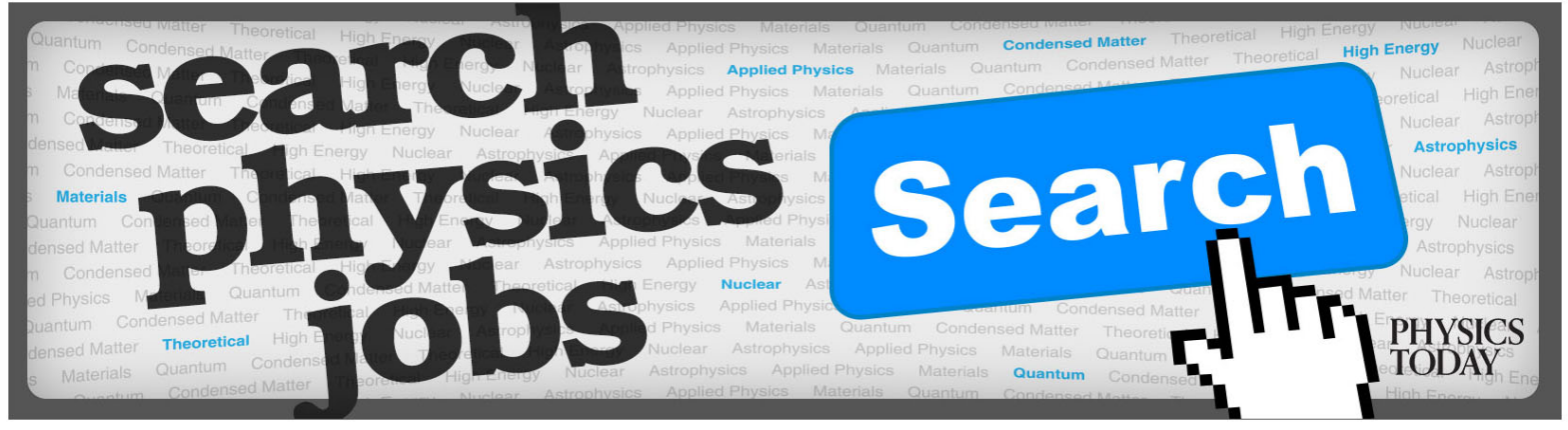




\title{
Analytic properties of the electromagnetic Green's function
}

\author{
Boris Gralak ${ }^{\mathrm{a})}$ \\ Aix Marseille Univ, CNRS, Centrale Marseille, Institut Fresnel, Marseille, France
}

(Received 21 December 2015; accepted 26 June 2017; published online 14 July 2017)

\begin{abstract}
Analytic properties of the electromagnetic Green's function are established and extended to a second complex frequency, introduced as a new degree of freedom, and to complex wavevectors. Next, Kramers-Kronig expressions for the inverse Helmholtz operator and the electromagnetic Green's function are derived by analogy with the permittivity. These Kramers-Kronig expressions are the starting point to propose a new method to obtain an expansion of Green's function on the leaky or quasinormal modes for open systems. This method is illustrated in the situation of a single dispersive layer. Finally, the second frequency introduced as a new degree of freedom is exploited to characterize non-dispersive systems. Published by AIP Publishing. [http://dx.doi.org/10.1063/1.4993199]
\end{abstract}

\section{INTRODUCTION}

The electromagnetic Green's function is a fundamental quantity in the analysis of systems described by macroscopic Maxwell's equations. ${ }^{1}$ It is defined from the inverse of the Helmholtz operator, ${ }^{2}$ which provides the electric field radiated by a current source. For a medium described by the electric permittivity $\varepsilon(\mathbf{x}, z)$ depending on the space variable $\mathbf{x} \in \mathbb{R}^{3}$ and the complex frequency $z$, the Helmholtz operator $\mathrm{H}_{e}(z)$ is given by

$$
\left[\mathrm{H}_{e}(z) \mathbf{E}\right](\mathbf{x}, z)=z^{2} \varepsilon(\mathbf{x}, z) \mu_{0} \mathbf{E}(\mathbf{x}, z)-\partial_{\mathbf{x}} \times \partial_{\mathbf{x}} \times \mathbf{E}(\mathbf{x}, z),
$$

where $\partial_{\mathbf{x}} \times$ is the curl operator and $\mu_{0}$ is the vacuum permeability. Then, the electromagnetic Green's (dyadic) function can be defined from the inverse of the Helmholtz operator by

$$
\left[\mathrm{H}_{e}(z)^{-1} \mathbf{S}\right](\mathbf{x})=\int_{\mathbb{R}^{3}} d \mathbf{y} \mathbf{G}_{e}(\mathbf{x}, \mathbf{y} ; z) \mathbf{S}(\mathbf{y}),
$$

where $\mathbf{S}(\mathbf{x})$ is proportional to a current source density.

The electromagnetic Green's function $\mathrm{G}_{e}(\mathbf{x}, \mathbf{y} ; z)$ is an analytic function in the upper half space of complex frequencies $z$ : this is a direct consequence of the causality principle and passivity. ${ }^{1,3}$ These properties can be used in time-dependent electromagnetic fields, ${ }^{4-6}$ for instance, defining analytic continuation in the plane of complex frequencies. They are also the starting point to derive Kramers-Kronig relations for the reflection and transmission coefficients of multilayers. ${ }^{7-10}$

In this paper, analytic properties of the electromagnetic Green's function $\mathrm{G}_{e}(\mathbf{x}, \mathbf{y} ; z)$ are rigorously established and extended, and are then exploited in different situations. These properties are derived using simple arguments based on the permittivity function and are then transposed to the inverse Helmholtz operator (1) and to Green's function (2). In Sec. II, basic properties of the permittivity $\varepsilon(\mathbf{x}, z)$ and the inverse Helmholtz operator are presented in a rigorous frame. This general approach is then used to extend the analytic properties of Green's function to a second complex frequency and to complex wavevectors. This second complex frequency appears as an additional degree of freedom, which is exploited in Sec. VI, to provide a rigorous characterization of non-dispersive systems satisfying the analytic properties and the causality requirement.

\footnotetext{
a)boris.gralak@fresnel.fr
} 
The electromagnetic Green's function is introduced in Sec. III, and in Sec. IV, an analogy with the permittivity is drawn to obtain Kramers-Kronig relations for the inverse Helmholtz operator and Green's function. These Kramers-Kronig relations are exploited in Sec. V as the starting point to extend to open systems the eigenmodes expansion established for a closed cavity. ${ }^{11}$ These relations are used as integral expressions, which unveil the leaky or quasinormal modes ${ }^{12-14}$ by analytic continuation. In particular, the proposed method provides new arguments showing the validity of the expansion on the quasinormal modes in the situation of a single dispersive layer. ${ }^{15,16}$

\section{ANALYTIC PROPERTIES OF THE HELMHOLTZ OPERATOR AND EXTENSION OF ITS DEFINITION}

\section{A. Maxwell's equations}

We start with Maxwell's equations in dielectric media. Let $\boldsymbol{E}(\mathbf{x}, t), \boldsymbol{H}(\mathbf{x}, t)$, and $\boldsymbol{P}(\mathbf{x}, t)$ be, respectively, the time-dependent electric, magnetic, and polarization fields. Then, equations of macroscopic electromagnetics ${ }^{1}$ are

$$
\begin{aligned}
& \varepsilon_{0} \partial_{t} \boldsymbol{E}(\mathbf{x}, t)+\partial_{t} \boldsymbol{P}(\mathbf{x}, t)=\partial_{\mathbf{x}} \times \boldsymbol{H}(\mathbf{x}, t)-\boldsymbol{J}(\mathbf{x}, t), \\
& \mu_{0} \partial_{t} \boldsymbol{H}(\mathbf{x}, t)=-\partial_{\mathbf{x}} \times \boldsymbol{E}(\mathbf{x}, t),
\end{aligned}
$$

where $\partial_{t}$ is the partial derivative with respect to time and $\boldsymbol{J}(\mathbf{x}, t)$ is the current source density. In addition, the electric field is related to the polarization through the constitutive equation

$$
\boldsymbol{P}(\mathbf{x}, t)=\int_{-\infty}^{t} d s \chi(\mathbf{x}, t-s) \boldsymbol{E}(\mathbf{x}, s),
$$

where $\chi(\mathbf{x}, t)$ is the electric susceptibility. A Fourier decomposition with respect to the time of the equations above leads to

$$
\begin{aligned}
& -i \omega \varepsilon(\mathbf{x}, \omega) \mathbf{E}(\mathbf{x}, \omega)=\partial_{\mathbf{x}} \times \mathbf{H}(\mathbf{x}, \omega)-\mathbf{J}(\mathbf{x}, \omega), \\
& -i \omega \mu_{0} \mathbf{H}(\mathbf{x}, \omega)=-\partial_{\mathbf{x}} \times \mathbf{E}(\mathbf{x}, \omega),
\end{aligned}
$$

where the dielectric permittivity is defined as

$$
\varepsilon(\mathbf{x}, \omega)-\varepsilon_{0}=\int_{0}^{\infty} d t \exp [i \omega t] \chi(\mathbf{x}, t) .
$$

Here, according to the causality principle, it has been used that the susceptibility $\chi(\mathbf{x}, t)$ vanishes for negative times, i.e., $\chi(\mathbf{x}, t)=0$ if $t<0$. Also, without loss of generality, it can be assumed that the susceptibility $\chi(\mathbf{x}, t)$ must be bounded by a linear function of the time $t$. Consequently, the expression above of the permittivity is actually well-defined if the real frequency is replaced by the complex frequency $z=\omega+i \eta$ with positive imaginary part $\operatorname{Im}(z)=\eta>0$. Under these conditions, its derivative with respect to the complex frequency is also well-defined since the function in the integral

$$
\frac{\partial \varepsilon}{\partial z}(\mathbf{x}, z)=\int_{0}^{\infty} d t(i t) \exp [i z t] \chi(\mathbf{x}, t)
$$

has exponential decay for $\operatorname{Im}(z)>0$. It follows that the permittivity $\varepsilon(\mathbf{X}, z)$ is an analytic function in the half plane of complex frequencies $z$ with positive imaginary part, which will be denominated by the "upper half plane" from now on. Finally, the susceptibility can be retrieved through the inverse Laplace transform

$$
\chi(\mathbf{x}, t)=\frac{1}{2 \pi} \int_{\Gamma_{\eta}} d z \exp [-i z t]\left[\varepsilon(\mathbf{x}, z)-\varepsilon_{0}\right],
$$

where $\Gamma_{\eta}$ is the horizontal line of complex numbers $z=\omega+i \eta$ with $\omega \in \mathbb{R}$ at a distance $\eta$ from the real axis.

The Helmholtz equation is obtained from the set of Eq. (5),

$$
\begin{aligned}
{\left[\mathrm{H}_{e}(\omega) \mathbf{E}\right](\mathbf{x}, \omega) } & =\omega^{2} \varepsilon(\mathbf{x}, \omega) \mu_{0} \mathbf{E}(\mathbf{x}, \omega)-\partial_{\mathbf{x}} \times \partial_{\mathbf{x}} \times \mathbf{E}(\mathbf{x}, \omega) \\
& =-i \omega \mu_{0} \mathbf{J}(\mathbf{x}, \omega) .
\end{aligned}
$$


Since the permittivity is well-defined in the upper half plane $\operatorname{Im}(z)>0$, the definition of the Helmholtz operator $\mathrm{H}_{e}(\omega)$ can be also extended to all complex frequencies $z$ with $\operatorname{Im}(z)>0$. It can be shown rigorously using the auxiliary field formalism ${ }^{17}$ that the operator $\mathrm{H}_{e}(z)$ is invertible and that the inverse $\mathrm{H}_{e}(z)^{-1}$ is analytic with respect to the complex frequency $z$ in the domain $\operatorname{Im}(z)>0$. The main arguments to show these properties are the following. ${ }^{17}$ Adding a new auxiliary field $\boldsymbol{A}(t)$ to the electromagnetic field to form the total vector field $F(t)=[\boldsymbol{E}(t), \boldsymbol{H}(t), \boldsymbol{A}(t)]^{T}$, the set of Maxwell's equations (3) [without the source $\boldsymbol{J}(\mathbf{x}, t)$ ] can be written as the unitary time-evolution equation $\partial_{t} F(t)$ $=-i \mathrm{~K} F(t)$, where $\mathrm{K}$ is a time-independent and self-adjoint operator. The inverse $[z-\mathrm{K}]^{-1}$ is then well-defined for all complex numbers $z$ with $\operatorname{Im}(z)>0$ and is moreover an analytic function of $z$. Next, the inverse $\mathrm{H}_{e}(z)^{-1}$ of the Helmholtz operator is retrieved by projecting the total field $F(z)$ on the electric fields $\mathbf{E}(z)$ and by using the Feshbach projection formula. ${ }^{18,19}$ Since the projector on electric fields is $z$-independent, the inverse of the Helmholtz operator has the same analytic properties as the inverse $[z-\mathrm{K}]^{-1}$.

In this paper, the analytical properties of the inverse Helmholtz operator and of Green's function are extended to other variables. It is proposed to obtain these new properties avoiding the construction of the auxiliary field formalism ${ }^{17}$ and rather using arguments based on the properties of the permittivity $\varepsilon(\mathbf{x}, z)$ which can be transposed directly to the Helmholtz operator and then to the electromagnetic Green's function.

\section{B. The permittivity}

Properties of the permittivity are derived in this subsection, in view to transpose them to the electromagnetic Green's function. The starting point is the generalized expression of Kramers-Kronig relations ${ }^{17,20}$ for the permittivity

$$
\varepsilon(\mathbf{x}, z)=\varepsilon_{0}-\int_{\mathbb{R}} d v \frac{\sigma(\mathbf{x}, v)}{z^{2}-v^{2}},
$$

where

$$
\sigma(\mathbf{x}, v)=\operatorname{Im} \frac{v\left[\varepsilon(\mathbf{x}, v)-\varepsilon_{0}\right]}{\pi} \geq 0 .
$$

Notice that the derivation of Kramers-Kronig relations like (10) is based on the asymptotic behaviors $\varepsilon(\mathbf{x}, z) \rightarrow \varepsilon_{0}$ occurring when the complex frequency $z$ tends to infinity. These behaviors are direct consequences of expression (6) where the real frequency $\omega$ is replaced by the complex one $z=\omega+i \eta$, showing that the permittivity is the Fourier transform of an integrable $\left[L^{1}(\mathbb{R})\right]$ function of the time. The relationship (11) between the function $\sigma(\mathbf{X}, v)$ and the imaginary part of the permittivity can be retrieved taking the $\operatorname{limit} \operatorname{Im}(z)=\eta \downarrow 0$ in (10). In this Eq. (11), it has been assumed that only passive media are considered. Indeed, under this assumption, the electromagnetic energy at any time $t$ must be lower than that at the initial time, and thus the permittivity must have a positive imaginary part: ${ }^{3}$ the function $\sigma(\mathbf{x}, v)$ is then positive as specified by Eq. (11). At the microscopic scale, this function corresponds to the oscillator strength ${ }^{21}$ (a positive quantity).

The passivity requirement (11) can be extended to all complex frequencies in the upper half plane: using that $\sigma(\mathbf{x}, v)=\sigma(\mathbf{x},-v)$, the expression (10) can be written as

$$
z\left[\varepsilon(\mathbf{x}, z)-\varepsilon_{0}\right]=-\int_{\mathbb{R}} d v \frac{\sigma(\mathbf{x}, v)}{z-v},
$$

which implies

$$
\operatorname{Im}\left\{z\left[\varepsilon(\mathbf{x}, z)-\varepsilon_{0}\right]\right\}=\operatorname{Im}(z) \int_{\mathbb{R}} d v \frac{\sigma(\mathbf{x}, v)}{|z-v|^{2}} \geq 0 .
$$

This passivity condition extended to complex frequency (13) is a key argument to obtain the analytic properties of Green's function in Secs. II C and II D.

In order to transpose the Kramers-Kronig expression from the permittivity to Green's function, additional inequalities are needed. The starting point is the relativity requirement implying that the derivative $\left(\partial_{t} \chi\right)(\mathbf{x}, t)$ of the susceptibility must be bounded. Indeed, this derivative corresponds to the microscopic currents, i.e., the charge velocities, and is then bounded by the light velocity in vacuum [and the susceptibility $\chi(\mathbf{x}, t)$ cannot increase faster than a linear function of the time 
as assumed before]. The derivative of the susceptibility is calculated using the Kramers-Kronig relation (12),

$$
\begin{aligned}
\left(\partial_{t} \chi\right)(\mathbf{x}, t) & =\frac{1}{2 \pi} \int_{\Gamma_{\eta}} d z \exp [-i z t](-i z)\left[\varepsilon(\mathbf{X}, z)-\varepsilon_{0}\right] \\
& =\frac{1}{2 \pi} \int_{\mathbb{R}} d v \sigma(\mathbf{x}, v) \int_{\Gamma_{\eta}} d z(i) \frac{\exp [-i z t]}{z-v} .
\end{aligned}
$$

The last integral is computed by closing the line $\Gamma_{\eta}$ by a semicircle in the upper (for $t<0$ ) or lower (for $t>0$ ) half spaces. It is retrieved that the susceptibility $\chi(\mathbf{x}, t)$ vanishes for $t<0$, and, for $t>0$,

$$
\left(\partial_{t} \chi\right)(\mathbf{x}, t)=\int_{\mathbb{R}} d v \sigma(\mathbf{x}, v) \cos [v t] .
$$

This implies in particular that the integral of the function $\sigma(\mathbf{x}, v)$ is finite since

$$
\int_{\mathbb{R}} d v \sigma(\mathbf{x}, v)=\left[\partial_{t} \chi\right]\left(\mathbf{x}, 0^{+}\right)<\infty .
$$

Also, it is obtained that, except at $t=0$, the function $\left[\partial_{t} \chi\right](\mathbf{x}, t)$ is continuous of $t$, and it can be checked that $\left[\partial_{t} \chi\right](\mathbf{x}, t)$ is bounded by $\left[\partial_{t} \chi\right]\left(\mathbf{x}, 0^{+}\right)$.

Finally, the asymptotic behavior of the permittivity can be specified when the modulus $|z|$ of the complex frequency tends to infinity (see Appendix A for a proof),

$$
z^{2}\left[\varepsilon(\mathbf{x}, z)-\varepsilon_{0}\right] \underset{|z| \rightarrow \infty}{\longrightarrow}-\left[\partial_{t} \chi\right]\left(\mathbf{x}, 0^{+}\right)
$$

The extension of this behavior in the limit where the real part $\omega$ of the complex frequency tends to infinity requires additional assumptions in the physics underlying the construction of the permittivity. Nevertheless, it can be established that the function $\varepsilon(\mathbf{x}, z)-\varepsilon_{0}$ is integrable $\left[L^{1}(\mathbb{R})\right]$ with respect to the real part of the frequency,

$$
\int_{\Gamma_{\eta}} d z\left|\varepsilon(\mathbf{X}, z)-\varepsilon_{0}\right| \leq \frac{\pi}{\eta}\left[\partial_{t} \chi\right]\left(\mathbf{x}, 0^{+}\right) .
$$

A proof is provided in Appendix B.

\section{Helmholtz operator and extension of its definition}

In order to rigorously define the inverse of the Helmholtz operator for complex frequencies $z$, the set of equations (5) is written as

$$
\left[\mathrm{M}_{0}(z)+\mathrm{V}(\mathbf{x}, z)\right] F(\mathbf{x})=S(\mathbf{x}),
$$

where $F(\mathbf{x})$ contains the electromagnetic field and $S(\mathbf{x})$ is the current source,

$$
F(\mathbf{x})=\left[\begin{array}{l}
\mathbf{E}(\mathbf{x}) \\
\mathbf{H}(\mathbf{x})
\end{array}\right], \quad S(\mathbf{x})=\left[\begin{array}{c}
-i \varepsilon_{0}^{-1} \mathbf{J}(\mathbf{x}) \\
0
\end{array}\right] .
$$

The operator $\mathrm{M}_{0}(z)$ corresponds to Maxwell's equations in vacuum and $\mathrm{V}(\mathbf{x}, z)$ contains the response of the material,

$$
\begin{aligned}
\mathrm{M}_{0}(z) & =\left[\begin{array}{lr}
z & i \varepsilon_{0}^{-1} \partial_{\mathbf{x}} \times \\
-i \mu_{0}^{-1} \partial_{\mathbf{x}} \times & z
\end{array}\right], \\
\mathrm{V}(\mathbf{x}, z) & =\left[\begin{array}{lr}
z\left\{\varepsilon(\mathbf{x}, z) / \varepsilon_{0}-1\right\} & 0 \\
0 & 0
\end{array}\right] .
\end{aligned}
$$

Let $\langle\cdot, \cdot\rangle$ be the standard inner product in the Hilbert space of square integrable electromagnetic fields,

$$
\left\langle F_{1}, F_{2}\right\rangle=\int_{\mathbb{R}^{3}} d \mathbf{x}\left[\varepsilon_{0} \overline{\mathbf{E}_{1}(\mathbf{x})} \cdot \mathbf{E}_{2}(\mathbf{x})+\mu_{0} \overline{\mathbf{H}_{1}(\mathbf{x})} \cdot \mathbf{H}_{2}(\mathbf{x})\right] .
$$


If for all fields $F$ there exists a constant $\alpha>0$ such that

$$
\left|\left\langle F,\left[\mathrm{M}_{0}(z)+\mathrm{V}(\mathbf{x}, z)\right] F\right\rangle\right| \geq \alpha\langle F, F\rangle,
$$

then the operator $\left[\mathrm{M}_{0}(z)+\mathrm{V}(\mathbf{x}, z)\right]$ is invertible, and its inverse is bounded by $\alpha^{-1}$. Using that the curl is self-adjoint, the following relationship is obtained:

$$
\begin{aligned}
\operatorname{Im}\left\langle F, \mathrm{M}_{0}(z) F\right\rangle & =\operatorname{Im}(z) \int_{\mathbb{R}^{3}} d \mathbf{x}\left[\varepsilon_{0}|\mathbf{E}(\mathbf{x})|^{2}+\mu_{0}|\mathbf{H}(\mathbf{x})|^{2}\right] \\
& =\operatorname{Im}(z)\langle F, F\rangle .
\end{aligned}
$$

The term with $\mathrm{V}(\mathbf{x}, z)$ containing the relative permittivity is estimated using the passivity requirement extended to the complex plane (13),

$$
\operatorname{Im}\langle F, \mathrm{~V}(\mathbf{x}, z) F\rangle=\int_{\mathbb{R}^{3}} d \mathbf{x}|\mathbf{E}(\mathbf{x})|^{2} \operatorname{Im}\left\{z\left[\varepsilon(\mathbf{x}, z)-\varepsilon_{0}\right]\right\} \geq 0
$$

The combination of the two equations leads to

$$
\left|\left\langle F,\left[\mathrm{M}_{0}(z)+\mathrm{V}(\mathbf{x}, z)\right] F\right\rangle\right| \geq \operatorname{Im}(z)\langle F, F\rangle,
$$

which implies that the inverse $\left[\mathrm{M}_{0}(z)+\mathrm{V}(\mathbf{x}, z)\right]^{-1}$ is well-defined for $\operatorname{Im}(z)>0$ and bounded by $\alpha^{-1}=1 / \operatorname{Im}(z)$. In addition, this inverse is an analytic function of the complex frequency $z$ for $\operatorname{Im}(z)>0$ as well as the permittivity function $\varepsilon(\mathbf{x}, z)$ in $\mathrm{V}(\mathbf{x}, z)$, and $\mathrm{M}_{0}(z)$. The analyticity property can be also inferred using the first resolvent formula, ${ }^{22}$ as shown in Appendix $\mathrm{C}$.

The inverse Helmholtz operator can be retrieved from the well-defined inverse $\left[\mathrm{M}_{0}(z)+\mathrm{V}(\mathbf{x}, z)\right]^{-1}$. It is obtained using the projector on electric fields $\mathrm{P}$, defined by $\mathrm{PF}(\mathbf{x})=\mathbf{E}(\mathbf{x})$. Then, Eq. (19) yields

$$
\begin{aligned}
\mathbf{E}(\mathbf{x})=\mathrm{P} F(\mathbf{x}) & =\mathrm{P}\left[\mathrm{M}_{0}(z)+\mathrm{V}(\mathbf{x}, z)\right]^{-1} \mathrm{~S}(\mathbf{x}) \\
& =\mathrm{P}\left[\mathrm{M}_{0}(z)+\mathrm{V}(\mathbf{x}, z)\right]^{-1} \mathrm{P} S(\mathbf{x}),
\end{aligned}
$$

since, according to (20), $S(\mathbf{x})=\mathrm{PS}(\mathbf{x})$ with the single "electric component" $-i \varepsilon_{0}^{-1} \mathbf{J}(\mathbf{x})$. Equation (9) implies that the inverse Helmholtz operator is given by $\mathbf{E}(\mathbf{x})=-i z \mu_{0} \mathrm{H}_{e}(z)^{-1} \mathbf{J}(\mathbf{x})$, and the comparison with the above expression provides

$$
\mathrm{H}_{e}(z)^{-1}=\frac{1}{z \mu_{0} \varepsilon_{0}} \mathrm{P}\left[\mathrm{M}_{0}(z)+\mathrm{V}(\mathbf{x}, z)\right]^{-1} \mathrm{P} .
$$

This expression shows that all the properties of the inverse $\left[\mathrm{M}_{0}(z)+\mathrm{V}(\mathbf{x}, z)\right]^{-1}$ can be directly transposed to the inverse Helmholtz operator. In particular, the inverse Helmholtz operator is bounded by

$$
\left\|\mathrm{H}_{e}(z)^{-1}\right\| \leq \frac{1}{|z| \varepsilon_{0} \mu_{0} \operatorname{Im}(z)} .
$$

It is stressed that the bound $\alpha=\operatorname{Im}(z)$ in (26) is governed by the imaginary part of $z$ in $\mathrm{M}_{0}(z)$ only and thus is independent of the complex number $z$ in $\mathrm{V}(\mathbf{x}, z)$, hence there is a possibility to independently consider the two complex frequencies in $\mathrm{M}_{0}(z)$ and $\mathrm{V}(\mathbf{x}, z)$. This remark leads to the following result: the inverse

$$
\left[\mathrm{M}_{0}(z)+\mathrm{V}(\mathbf{x}, \xi)\right]^{-1} \leq[\operatorname{Im}(z)]^{-1}
$$

exists and is analytic with respect to both complex frequencies $z$ and $\xi$ in the domain $\operatorname{Im}(z)>0$ and $\operatorname{Im}(\xi)>0$. Also, power series expansions like (C3) can be independently stated for both variables $z$ and $\xi$. This property can be transposed to the inverse of a generalized version of the Helmholtz operator. The expression of this operator, denoted by $\mathrm{H}(z, \xi)$, can be obtained by replacing $\mathrm{V}(\mathbf{x}, z)$ by $\mathrm{V}(\mathbf{x}, \xi)$ and then by eliminating the magnetic field $\mathbf{H}(\mathbf{x})$ in Eq. (19),

$$
\mathrm{H}(z, \xi)=z^{2} \varepsilon_{0} \mu_{0}+z \mu_{0} \xi\left[\varepsilon(\mathbf{x}, \xi)-\varepsilon_{0}\right]-\partial_{\mathbf{x}} \times \partial_{\mathbf{x}} \times .
$$


A relation similar to (28) shows that the inverse of $\mathrm{H}(z, \xi)$ exists and is analytic of both complex variables $z$ and $\xi$ in the domain $\operatorname{Im}(z)>0$ and $\operatorname{Im}(\xi)>0$. In addition, it is bounded by

$$
\left\|\mathrm{H}(z, \xi)^{-1}\right\| \leq \frac{1}{|z| \varepsilon_{0} \mu_{0} \operatorname{Im}(z)} .
$$

This extended definition of the Helmholtz operator is used in Sec. VI to analyze non-dispersive systems.

\section{Analytic properties with respect to the wavevector}

In this section, it is assumed that a wavevector $\mathbf{k}$ can be defined, which requires for the geometry of the system to be invariant under a group of translations. The starting point is the expression of Maxwell's equations (21) introduced in Sec. II C. In case the group of translations is discrete (periodic structure), the curl operator becomes $[\nabla+i \mathbf{k}] \times$ after a Floquet-Bloch decomposition and, in case the group of translation is continuous (homogeneous structure), the curl operator becomes $i \mathbf{k} \times$ after a Fourier transform. The resulting free operator introduced in (21) becomes $\mathrm{M}_{0}(\mathbf{k}, z)$, while the potential $\mathrm{V}(\mathbf{x}, z)$ is left invariant. The wavevector $\mathbf{k}$ can have one, two, or three components if the geometry of the system is invariant under translations in one, two, or three dimensions, respectively. Let $\mathbf{k}^{\prime}$ and $\mathbf{k}^{\prime \prime}$ be the real and imaginary parts of the wavevector, $\mathbf{k}=\mathbf{k}^{\prime}+i \mathbf{k}^{\prime \prime}$. Then the "imaginary" part of the free operator $\mathrm{M}_{0}(\mathbf{k}, z)$ can be computed as follows:

$$
\frac{\mathrm{M}_{0}(\mathbf{k}, z)-\mathrm{M}_{0}(\mathbf{k}, z)^{\dagger}}{2 i}=\left[\begin{array}{lr}
\operatorname{Im}(z) & -\varepsilon_{0}^{-1} \mathbf{k}^{\prime \prime} \times \\
\mu_{0}^{-1} \mathbf{k}^{\prime \prime} \times & \operatorname{Im}(z)
\end{array}\right],
$$

where the symbol $\dagger$ indicates the adjoint. The eigenvalues of this matrix are

$$
\lambda_{0}=\operatorname{Im}(z), \quad \lambda_{ \pm}=\operatorname{Im}(z) \pm c k^{\prime \prime},
$$

where $c$ is the light velocity in vacuum $\left(1 / c^{2}=\varepsilon_{0} \mu_{0}\right)$, and $\left(k^{\prime \prime}\right)^{2}=\mathbf{k}^{\prime \prime} \cdot \mathbf{k}^{\prime \prime}$. Consequently, this imaginary part of the free operator is strictly positive as soon as

$$
\operatorname{Im}(z)>c\left|k^{\prime \prime}\right|>0 .
$$

Since the imaginary part of the potential $\mathrm{V}(\mathbf{x}, z)$ is also positive $(25)$, the inverse $\left[\mathrm{M}_{0}(\mathbf{k}, z)+\mathrm{V}(\mathbf{x}, z)\right]$ is well-defined and analytic in the domain $\operatorname{Im}(z)-c\left|k^{\prime \prime}\right|>0$ of complex frequencies $z$ and wavevectors $\mathbf{k}$. And it is straightforward to extend this property to the inverse $\left[\mathrm{M}_{0}(\mathbf{k}, z)+\mathrm{V}(\xi, z)\right]$ in the domain defined by (i) $\operatorname{Im}(z)-c\left|k^{\prime \prime}\right|>0$ and (ii) $\operatorname{Im}(\xi)>0$. As a remark, it is stressed that the analytic property of the inverse Helmholtz operator in the domain (35) is equivalent to the relativity requirement stating that the electromagnetic field cannot propagate faster than the light velocity in vacuum $c$. This equivalence can be shown using arguments inspired from the Paley-Wiener theorem. ${ }^{23}$

An analogous property has been used in Ref. 10 to derive new Kramers-Kronig relations for the reflection and transmission coefficients (via Green's function) in the case of multilayered stacks illuminated with incident angle $\theta \neq 0$. Indeed, in this situation, the square of the wavevector appears to be $\mathbf{k} \cdot \mathbf{k}=\left(z^{2} / c^{2}\right) \sin ^{2} \theta$, which always meets the requirement (35).

\section{THE ELECTROMAGNETIC GREEN'S FUNCTION}

The electromagnetic Green's function is related to the inverse Helmholtz operator as shown by Eq. (2). While the left side of the equation, $\mathrm{H}_{e}(z)^{-1} \mathbf{S}$, is well-defined, there is no argument ensuring the existence in electromagnetism of Green's function in the right side. Indeed, the existence of Green's function is usually the consequence of the compact or Hilbert-Schmidt nature of the corresponding inverse operator. ${ }^{22}$ However, this compact nature cannot be obtained in the case of electromagnetism, even by considering the difference ${ }^{24}$ of the original inverse $\left[\mathrm{M}_{0}(z)+\mathrm{V}(\mathbf{x}, z)\right]^{-1}$ with the free inverse $\mathrm{M}_{0}(z)^{-1}$. This lack of compactness in electromagnetism results from the presence of the "static" modes which generate a "Dirac" singularity in Green's function (see Ref. 25 for investigations on the singularity). In practice, for square integrable functions $\phi$ and $\psi$, it is always possible to define coefficients like

$$
\left\langle\phi, \mathrm{H}_{e}(z)^{-1} \psi\right\rangle_{e}=\int_{\mathbb{R}^{3}} d \mathbf{x} \overline{\phi(\mathbf{x})}\left[\mathrm{H}_{e}(z)^{-1} \psi\right](\mathbf{x})
$$


corresponding to

$$
\int_{\mathbb{R}^{3}} d \mathbf{x} \int_{\mathbb{R}^{3}} d \mathbf{y} \overline{\phi(\mathbf{x})} \mathrm{G}_{e}(\mathbf{x}, \mathbf{y} ; z) \psi(\mathbf{y}) .
$$

Here, the bar indicates the complex conjugated and the brackets $\langle\cdot, \cdot\rangle_{e}$ denote the standard inner product (36) for the solely electric fields. The functions $\phi$ and $\psi$ can be the elements of an orthonormal basis $\left\{\phi_{p}(\mathbf{x})\right\}$ of the square integrable functions. Then, the coefficients $\left\langle\phi_{p}, \mathrm{H}_{e}(z)^{-1} \phi_{q}\right\rangle_{e}$ can be used to define "formally"

$$
\mathrm{G}_{e}(\mathbf{x}, \mathbf{y} ; z)=\sum_{n, m}\left\langle\phi_{p}, \mathrm{H}_{e}(z)^{-1} \phi_{q}\right\rangle_{e} \phi_{p}(\mathbf{x}) \otimes \overline{\phi_{q}(\mathbf{y})},
$$

where the symbol $\otimes$ means that the tensor product is considered. Notice that this definition is only "formal" because there is no argument ensuring that the convergence of the sum in the case $\mathrm{H}_{e}(z)^{-1}$ is not Hilbert-Schmidt. The functions $\phi$ and $\psi$ in (36) and (37) can be also chosen to approach the identity. Let the function $\phi_{a}$ be defined by $\phi_{a}(\mathbf{x})=(3 / 4 \pi) a^{-3}$ if $|\mathbf{x}| \leq a$ and $\phi_{a}(\mathbf{x})=0$ if $|\mathbf{x}| \geq a$ in order to approach the Dirac function $\delta(\mathbf{x})$ when $a \downarrow 0$. Then, for $a$ small enough, $\phi_{\mathbf{x}_{0}}(\mathbf{x})=\phi_{a}\left(\mathbf{x}-\mathbf{x}_{0}\right) \approx \delta\left(\mathbf{x}-\mathbf{x}_{0}\right)$ and $\phi_{\mathbf{y}_{0}}(\mathbf{y})=\phi_{a}\left(\mathbf{y}-\mathbf{y}_{0}\right) \approx \delta\left(\mathbf{y}-\mathbf{y}_{0}\right)$, and the coefficient $\left\langle\phi_{\mathbf{x}_{0}}, \mathrm{H}_{e}(z)^{-1} \phi_{\mathbf{y}_{0}}\right\rangle_{e}$ can approach Green's function

$$
\begin{aligned}
& \left\langle\phi_{\mathbf{x}_{0}}, \mathrm{H}_{e}(z)^{-1} \phi_{\mathbf{y}_{0}}\right\rangle_{e} \\
& \quad=\int_{\mathbb{R}^{3}} d \mathbf{x} \int_{\mathbb{R}^{3}} d \mathbf{y} \phi_{a}\left(\mathbf{x}-\mathbf{x}_{0}\right) \mathrm{G}_{e}(\mathbf{x}, \mathbf{y} ; z) \phi_{a}\left(\mathbf{y}-\mathbf{y}_{0}\right) \\
& \quad \approx \mathrm{G}_{e}\left(\mathbf{x}_{0}, \mathbf{y}_{0} ; z\right) .
\end{aligned}
$$

Here, it is stressed that nothing proves that the coefficients like (36) and (39) exist at the limit $a \downarrow 0$ since the function $\phi_{a}$ is not square integrable at this limit. Thus the coefficient $\left\langle\phi_{\mathbf{x}_{0}}, \mathrm{H}_{e}(z)^{-1} \phi_{\mathbf{y}_{0}}\right\rangle_{e}$ only allows us to address an approximation of Green's function $\mathrm{G}_{e}\left(\mathbf{x}_{0}, \mathbf{y}_{0} ; z\right)$.

According to these arguments above, it is assumed that the electromagnetic Green's function can be defined and that its properties can be established from the coefficients $\left\langle\phi, \mathrm{H}_{e}(z)^{-1} \psi\right\rangle_{e}$. Under this assumption, all the analytic properties of $\mathrm{H}_{e}(z)^{-1}$ and $\mathrm{H}(z, \xi)^{-1}$ can be directly transposed to coefficients $\left\langle\phi, \mathrm{H}_{e}(z)^{-1} \psi\right\rangle_{e}$ and $\left\langle\phi, \mathrm{H}(z, \xi)^{-1} \psi\right\rangle_{e}$ since all the arguments presented in Subsection II C and Appendix C (C3) can be directly used with these coefficients. Hence, all the following results established for the inverse Helmholtz operator remain true for the electromagnetic Green's function.

The asymptotic behavior (17) and the integrable nature (18) of the permittivity can be also transposed to the inverse Helmholtz operator and to Green's function. This can be established for the Helmholtz operator writing the difference

$$
\mathrm{H}_{e}(z)^{-1}-\mathrm{H}_{0}(z)^{-1}=-\mathrm{H}_{0}(z)^{-1} z^{2} \mu_{0}\left[\varepsilon(\mathbf{x}, z)-\varepsilon_{0}\right] \mathrm{H}_{e}(z)^{-1} .
$$

Then, using the bound (29) and the asymptotic behavior (17), it is obtained that

$$
\left\|\mathrm{H}_{e}(z)^{-1}-\mathrm{H}_{0}(z)^{-1}\right\| \underset{|z| \rightarrow \infty}{\leq} \frac{\left\|\partial_{t} \chi\right\|_{\infty}}{|z|^{2} \varepsilon_{0}^{2} \mu_{0}^{2} \operatorname{Im}(z)^{2}},
$$

where $\left\|\partial_{t} \chi\right\|_{\infty}$ is the upper bound of the function $\left[\partial_{t} \chi\right]\left(\mathbf{x}, 0^{+}\right)$. In the case of the limit where the real part $\omega$ of the complex frequency tends to infinity (while the imaginary part is fixed), the integrable nature (18) of the permittivity and bound (29) implies

$$
\int_{\Gamma_{\eta}} d z\left\|\mathrm{H}_{e}(z)^{-1}-\mathrm{H}_{0}(z)^{-1}\right\| \leq \frac{\pi}{\eta} \frac{\left\|\partial_{t} \chi\right\|_{\infty}}{\varepsilon_{0}^{2} \mu_{0}^{2} \eta^{2}} .
$$

These properties and asymptotic behaviors will be used in Sec. IV to derive a version of KramersKronig relations for the inverse $\mathrm{H}_{e}(z)^{-1}$ and the electromagnetic Green's function.

\section{KRAMERS-KRONIG RELATIONS FOR THE ELECTROMAGNETIC GREEN'S FUNCTION}

The Kramers-Kronig relations can be applied to all functions derived from a causal signal. It is generally used in electromagnetism to analyze the permittivity, the permeability, or the optical 
index. ${ }^{1,3}$ A new version of Kramers-Kronig relations for complex frequencies, given by Eq. (10), has recently been proposed. ${ }^{17,20}$ This version shows that the general expression of the permittivity is a continuous superposition of elementary resonances given by the elastically bound electron model: it extends the classical Drude-Lorentz expression ${ }^{1}$ of the permittivity and also its quantum mechanical justification based on the electric dipole approximation. ${ }^{21}$ In particular, the continuous superposition of resonances in (10) describes a regime with absorption, while the quantum mechanics model is reduced to a discrete superposition of resonances without absorption.

In this section, it is proposed to obtain the version of Kramers-Kronig relations with complex frequencies for the electromagnetic Green's function or, equivalently, for the inverse operator $\mathrm{H}_{e}(z)^{-1}$. The objective is to transpose all the properties of the permittivity and to make it possible to use all the knowledge on permittivity $\varepsilon(\mathbf{x}, z)$ for the electromagnetic Green's function.

The inverse operators $\mathrm{H}_{e}(z)^{-1}$ and $\mathrm{H}_{0}(z)^{-1}$ are expected to behave similarly as the permittivities $\varepsilon(\mathbf{x}, z)$ and $\varepsilon_{0}$, respectively. Thus the following difference is considered:

$$
\mathrm{R}(z)=\mathrm{H}_{e}(z)^{-1}-\mathrm{H}_{0}(z)^{-1} \text {. }
$$

First, it is noticed that, as well as the inverses $\mathrm{H}_{e}(z)^{-1}$ and $\mathrm{H}_{0}(z)^{-1}$, the adjoint operator of $\mathrm{R}(z)$ is

$$
[\mathrm{R}(z)]^{\dagger}=\mathrm{R}(-\bar{z})
$$

which is related to $\overline{\varepsilon(z)}=\varepsilon(z)^{\dagger}=\varepsilon(-\bar{z})$. Next, let the operator $\mathrm{X}(t)$ be defined by

$$
\mathrm{X}(t)=\int_{\Gamma_{\eta}} d z \exp [-i z t] \mathrm{R}(z),
$$

where, as previously, $\Gamma_{\eta}$ is the horizontal line parallel to the real axis at a distance $\eta$ (i.e., the complex numbers $z=\omega+i \eta$ with fixed imaginary part $\eta>0$ ). It is stressed that this integral is well defined since, thanks to (42), $\mathrm{R}(z)$ is integrable. Also, it implies that $\mathrm{X}(t)$ is expressed from the Fourier transform of an integrable function, and thus $\mathrm{X}(t)$ is continuous of $t$. The integral expression of $\mathrm{X}(t)$ is independent of $\eta$ thanks to the analytic nature of the function under the integral. The operator $\mathrm{X}(t)$ is self-adjoint since, for $z=\omega+i \eta$,

$$
\mathrm{X}(t)^{\dagger}=\int_{\Gamma_{\eta}} d z \exp [i \bar{z} t] \mathrm{R}(-\bar{z})=\int_{\Gamma_{\eta}} d z \exp [-i z t] \mathrm{R}(z)
$$

where (44) has been used and the change $\omega \rightarrow-\omega$ has been performed to obtain the last expression. In addition, it can be checked that $\mathrm{X}(t)$ vanishes for negative times. Indeed, for $t<0$, integral (45) can be computed by closing the line $\Gamma_{\eta}$ by a semicircle with infinite radius in the upper half plane. With the asymptotic behavior (41), the integral on this semi-circle vanishes and, since all the functions are analytic, it is found that $\mathrm{X}(t)=0$ if $t<0$. Hence $\mathrm{X}(t)=\theta(t) \mathrm{X}(t)$, where $\theta(t)$ is the Heaviside function: $\theta(t)=0$ for $t<0$ and $\theta(t)=1$ for $t>0$. The quantity $\mathbf{X}(t)$ is then analogous to the real susceptibility $\chi(\mathbf{X}, t)$ : it is self-adjoint, symmetric, and it is associated with causality principle. And the difference $\mathrm{R}(z)$ is, as expected, analogous to $\varepsilon(\mathbf{x}, z)-\varepsilon_{0}$.

Next, it is possible for $\operatorname{Im}(z)=\eta>0$ to express the difference $\mathrm{R}(z)$ as the inverse Laplace transform of Eq. (45),

$$
\mathrm{R}(z)=\frac{1}{2 \pi} \int_{0}^{\infty} d t \exp [i z t] \mathrm{X}(t) .
$$

Here, the operator $\mathrm{R}(z)$ appears as the Laplace transform of $\mathrm{X}(t)=\theta(t) \mathrm{X}(t)$. It follows that $\mathrm{R}(z)$ can be written as the convolution of itself with the Laplace transform of $\theta(t)$, i.e., with $1 / z$. Let $\xi=v+i \zeta$ be a complex number with positive imaginary part $\zeta$ such that $\operatorname{Im}(z)=\eta>\zeta>0$. Then, a convolution expression of $\mathrm{R}(z)$ is

$$
\mathrm{R}(z)=-\frac{1}{2 i \pi} \int_{\Gamma_{\zeta}} d \xi \frac{\mathrm{R}(\xi)}{z-\xi}
$$

Notice that this expression can be also obtained using Cauchy's integral formula. Thanks to the analyticity with respect to the variable $\xi$, the integration line $\Gamma_{\zeta}$ above can be shifted to the real axis,

$$
\mathrm{R}(z)=-\frac{1}{2 i \pi} \int_{\mathbb{R}} d v \frac{\mathrm{R}(v)}{z-v} .
$$


Finally, using the odd or even nature of the symmetric or antisymmetric parts of $\mathrm{R}(z)$, expressions similar to (10) and (12) can be established (see Appendix D). Let $\mathrm{D}(v)$ be defined by

$$
\mathrm{D}(v)=\lim _{\zeta \downarrow 0} \mathrm{D}(v+i \zeta), \quad \mathrm{D}(\xi)=\frac{1}{2 i \pi}[\xi \mathrm{R}(\xi)-\bar{\xi} \mathrm{R}(-\bar{\xi})],
$$

where $\xi=v+i \zeta$. It is the symmetric part of $\xi \mathrm{R}(\xi)$ with respect to $v$, and it is then left invariant when $v$ is changed in $-v$. It implies the following expressions (see Appendix D):

$$
\begin{aligned}
& \mathrm{R}(z)=-\int_{\mathbb{R}} d v \frac{\mathrm{D}(v)}{z^{2}-v^{2}} \quad \operatorname{Im} z>0, \\
& z \mathrm{R}(z)=-\int_{\mathbb{R}} d v \frac{\mathrm{D}(\xi)}{z-\xi} \quad \operatorname{Im} z>\operatorname{Im} \xi \geq 0 .
\end{aligned}
$$

These equations define Kramers-Kronig expressions for the inverse Helmholtz operator. The first line of the equation above implies for the coefficients,

$$
\left\langle\phi, \mathrm{H}_{e}(z)^{-1} \psi\right\rangle=\left\langle\phi, \mathrm{H}_{0}(z)^{-1} \psi\right\rangle-\int_{\mathbb{R}} d v \frac{\langle\phi, \mathrm{D}(v) \psi\rangle}{z^{2}-v^{2}},
$$

and for the Green's function,

$$
\mathrm{G}_{e}(\mathbf{x}, \mathbf{y} ; z)=\mathrm{G}_{0}(\mathbf{x}, \mathbf{y} ; z)-\int_{\mathbb{R}} d v \frac{\rho(\mathbf{x}, \mathbf{y} ; v)}{z^{2}-v^{2}},
$$

where

$$
\rho(\mathbf{x}, \mathbf{y} ; v)=\frac{v \operatorname{Im}\left[\mathbf{G}_{e}(\mathbf{x}, \mathbf{y} ; v)-\mathbf{G}_{0}(\mathbf{x}, \mathbf{y} ; v)\right]}{\pi}
$$

is related to the imaginary part of the relative Green's function [modulo of the free Green's function $\left.\mathrm{G}_{0}(\mathbf{x}, \mathbf{y} ; v)\right]$. It is stressed that the quantity $\rho(\mathbf{x}, \mathbf{y} ; v)$ is closely related to the local density of states, which is proportional to the trace of $\rho(\mathbf{x}, \mathbf{x} ; v)$ [modulo of the density of states in vacuum].

\section{EIGENMODES EXPANSION OF THE GREEN'S FUNCTION}

The Kramers-Kronig relations obtained in Sec. IV can be exploited to extend to open systems the classical eigenmodes expansion of Green's function ${ }^{11}$ in a closed cavity. This extension to open systems uses a development of the inverse Helmholtz operator and Green's function on the leaky or quasinormal modes. ${ }^{12-16}$ Indeed, the Kramers-Kronig relations provide integral expressions like (48) and (51), which can be used in the most general situations (including open systems) where the causality principle applies with frequency dispersion and absorption.

The idea to exploit is to deform the integration line $\Gamma_{\zeta}$ (which can be the real axis $\mathbb{R}$ ) in (48) and (51)... in order to obtain an expression of the inverse Helmholtz operator and of Green's function reduced to a sum of isolated quasinormal modes (see Fig. 1). This technique is illustrated in a simple situation in Subsections V A and V B.

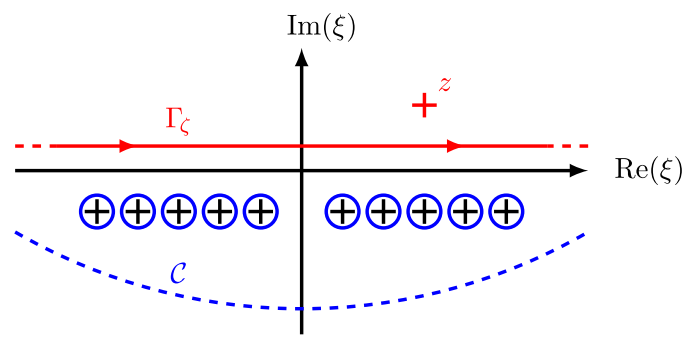

FIG. 1. The original integration contour $\Gamma_{\zeta}$ in red is the Kramers-Kronig expression (48) of the inverse R(z). After deformation, this contour becomes the blue path $\mathcal{C}$ and the blue circles around the isolated resonances (black crosses) of the system. 


\section{A. Green's function of a dispersive and absorptive homogeneous layer}

The one-dimensional scalar Helmholtz equation is considered,

$$
\left[\mathrm{H}_{e}(z) E\right](x, z)=z^{2} \varepsilon(x, z) \mu_{0} E(x, z)+\frac{d^{2} E}{d x^{2}}(x, z),
$$

where $x$ is the space variable in $\mathbb{R}$. This equation can describe a system made of the scalar field amplitude $E(x, z)$ and of a dispersive and absorptive homogeneous layer in vacuum (see Fig. 2). The permittivity $\varepsilon(X, z)$ is defined as

$$
\begin{aligned}
x>d: \varepsilon(x, z) & =\varepsilon_{0}, \\
d>x>-d: \varepsilon(x, z) & =\varepsilon_{0} \varepsilon_{1}(z), \\
-d>x: \varepsilon(x, z) & =\varepsilon_{0} .
\end{aligned}
$$

It is assumed that the relative permittivity $\varepsilon_{1}(z)$ is well described by a finite sum of Drude-Lorentz resonances

$$
\varepsilon_{1}(z)=1-\sum_{n=0}^{N} \frac{\sigma_{n}}{z^{2}+i \gamma_{n} z-\omega_{n}^{2}},
$$

where $\sigma_{n}, \gamma_{n}$, and $\omega_{n}$ are real and positive parameters. Let $\mathrm{G}_{e}(x, y ; z)$ be the total Green's function associated with the Helmholtz equation (55). The expression of its free part $\mathrm{G}_{0}(x-y ; z)$, defined taking $\varepsilon(X, z)=\varepsilon_{0}$ in (55), is proportional to

$$
\mathrm{G}_{0}(x-y ; z) \propto \exp [i z|x-y| / c] .
$$

Without loss of generality, the point source in Green's function is fixed at $y>d$ as shown in Fig. 2 . Then, the part of Green's function diffracted by the layer, denoted by $\mathrm{G}_{1}(x, y ; z)$, is given (outside the layer) by

$$
\begin{aligned}
x>d: \mathrm{G}_{1}(x, y ; z) & =\exp [i z y / c] r(z) \exp [i z x / c], \\
-d>x: \mathrm{G}_{1}(x, y ; z) & =\exp [i z y / c] t(z) \exp [-i z x / c],
\end{aligned}
$$

where $r(z)$ and $t(z)$ are the reflection and transmission coefficients (see Fig. 2). The expressions of these coefficients are 26,27

$$
\begin{aligned}
& r(z)=\frac{r_{0}(z)\left\{1-\exp \left[4 i z \sqrt{\varepsilon_{1}(z)} d / c\right]\right\}}{1-r_{0}(z)^{2} \exp \left[4 i z \sqrt{\varepsilon_{1}(z)} d / c\right]} \exp [-2 i z d / c], \\
& t(z)=\frac{\left[1-r_{0}(z)\right]^{2} \exp \left[2 i z \sqrt{\varepsilon_{1}(z)} d / c\right]}{1-r_{0}(z)^{2} \exp \left[4 i z \sqrt{\varepsilon_{1}(z)} d / c\right]} \exp [-2 i z d / c],
\end{aligned}
$$

where

$$
r_{0}(z)=\frac{1-\sqrt{\varepsilon_{1}(z)}}{1+\sqrt{\varepsilon_{1}(z)}}
$$

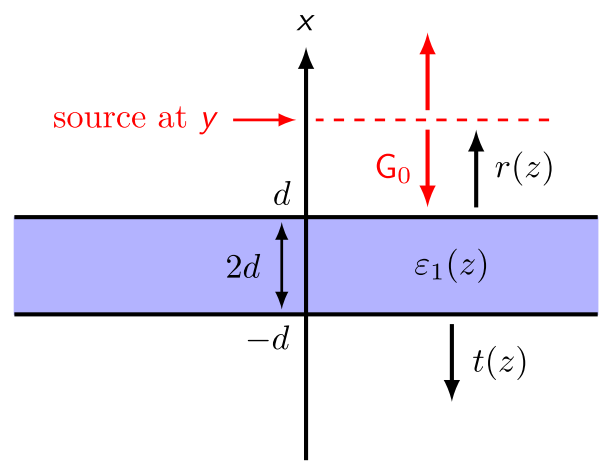

FIG. 2. The considered dispersive and absorptive homogeneous layer of thickness $2 d$. The point source in Green's function is located at $y>d$. 
is the reflection coefficient at the interface separating the vacuum from the dielectric of relative permittivity $\varepsilon_{1}(z)$.

A remarkable property is that the coefficients $r(z)$ and $t(z)$ are even functions of the square root $\sqrt{\varepsilon_{1}(z)}$, as shown in Refs. 26 and 27. This implies that the set of singularities in $r(z)$ and $t(z)$ reduces to isolated poles, leading to the absence of branch points and branch cuts (with respect to the complex frequency $z$ ) in the function $\mathrm{G}_{1}(x, y ; z)$. It is stressed that the expression of the function $\mathrm{G}_{1}(x, y ; z)$ inside the homogeneous layer is also free from branch cuts, and thus the following statements remain valid for Green's function in the whole space of $(x, y)$.

\section{B. Eigenmodes expansion of Green's function}

The starting point is the integral expression of the function $\mathrm{G}_{1}(x, y ; z)$ derived from the KramersKronig equation (48),

$$
\mathrm{G}_{1}(x, y ; z)=-\frac{1}{2 i \pi} \int_{\Gamma_{\zeta}} d \xi \frac{\mathrm{G}_{1}(x, y ; \xi)}{z-\xi} .
$$

Let $z_{p}$ be the poles of the reflection and transmission coefficients, and $r^{(p)}$ and $t^{(p)}$ be the associated residues (see Appendix E)

$$
\begin{aligned}
r^{(p)} & =\lim _{z \rightarrow z_{p}}\left(z-z_{p}\right) r(z)= \pm i c /(4 d), \\
t^{(p)} & =\lim _{z \rightarrow z_{p}}\left(z-z_{p}\right) t(z)=\mp i c /(4 d) .
\end{aligned}
$$

These poles can be estimated theoretically in the present case of the dispersive layer. ${ }^{27}$ In more complex situations, numerical methods can be used to compute these poles ${ }^{28,29}$ with the possibility to implement linearization techniques of the frequency dispersion. ${ }^{30,31}$ The residues $\mathrm{G}_{1}^{(p)}(x, y)$ of Green's function $\mathrm{G}_{1}(x, y ; z)$, corresponding to the poles $z_{p}$, are given by

$$
\begin{aligned}
x>d: \mathrm{G}_{1}^{(p)}(x, y) & =\exp \left[i z_{p} y / c\right] r^{(p)} \exp \left[i z_{p} x / c\right], \\
-d>x: \mathrm{G}_{1}^{(p)}(x, y) & =\exp \left[i z_{p} y / c\right] t^{(p)} \exp \left[-i z_{p} x / c\right] .
\end{aligned}
$$

As shown on Fig. 1, the integration line $\Gamma_{\zeta}$ in (62) can be deformed in a path $\mathcal{C}$ in the lower half plane, below the set of isolated poles, which reveals the contributions of the poles $z_{p}$ in the expression of $\mathrm{G}_{1}$

$$
\mathrm{G}_{1}(x, y ; z)=\sum_{p} \frac{\mathrm{G}_{1}^{(p)}(x, y)}{z-z_{p}}+\frac{1}{2 i \pi} \int_{\mathcal{C}} d \xi \frac{\mathrm{G}_{1}(x, y ; \xi)}{z-\xi} .
$$

At this stage, in order to obtain an expansion on the solely modes associated with the poles $z_{p}$, it is enough to show that the resulting integration over the path $\mathcal{C}$ vanishes. However, a severe difficulty appears in this situation with the exponentially growing nature of Green's function $\mathrm{G}_{1}(x, y ; \xi)$ when the complex frequency $\xi$ has negative imaginary part. A solution is to introduce perfectly matched layers ${ }^{13,14,32}$ in order to damp this exponential growth and to allow the analytic continuation of Green's function for $\xi$ with a negative imaginary part on the path $\mathcal{C}$. These perfectly matched layers can be defined by the dilation of coordinates

$$
x \longrightarrow x_{\alpha}=\alpha x, \quad y \longrightarrow y_{\alpha}=\alpha y,
$$

where $\alpha$ takes complex values. Hence the following Green's function is introduced:

$$
\mathrm{G}_{1}(x, y ; \xi) \longrightarrow \mathrm{G}_{\alpha}(x, y ; \xi)=\mathrm{G}_{1}(\alpha x, \alpha y ; \xi) .
$$

Then, for dilation coefficient $\alpha$ with a positive imaginary part (or a positive argument), the exponential functions in (59) become $\exp [i \alpha \xi y / c]$ and $\exp [ \pm i \alpha \xi X / c]$ and they decay with respect to the space variable. Finally, the behaviour of the coefficients $r(\xi)$ and $t(\xi)$ in the expression of $\mathrm{G}_{1}(x, y ; \xi)$ is analyzed in Appendix E. This analysis shows that, except in a strip around the curve $\operatorname{Im}(\xi)=-(c / d) \ln [\operatorname{Re}(\xi)]$ in which the poles $z_{p}$ are located, the coefficients $r(\xi)$ and $t(\xi)$ tend to 0 
or are bounded when the frequency $\xi$ tends to infinity. Let $\xi=|\xi| e^{i \phi}$; the integral in (65) can be written as

$$
\begin{aligned}
\int_{\mathcal{C}} d \xi \frac{\mathrm{G}_{1}(x, y ; \xi)}{z-\xi} & =\int_{\pi}^{2 \pi} d \phi i \xi \frac{\mathrm{G}_{1}(x, y ; \xi)}{z-\xi} \\
& \approx-i \int_{\pi}^{2 \pi} d \phi \mathrm{G}_{1}(x, y ; \xi) .
\end{aligned}
$$

First, the following part of the integral is considered:

$$
I_{\delta}=\int_{\pi}^{\pi+\delta} d \phi \mathrm{G}_{1}(x, y ; \xi)+\int_{2 \pi-\delta}^{2 \pi} d \phi \mathrm{G}_{1}(x, y ; \xi) .
$$

The contribution of the strip around the poles (see Appendix E) is the discrete sum in expression (65). Outside this strip, the coefficients $r(\xi)$ and $t(\xi)$ are bounded and thus also the function $\mathrm{G}_{1}(x, y ; \xi)$. Hence the integral $I_{\delta}$ can be made arbitrarily small for $\delta$ small enough. In the second part of the integral

$$
\int_{\pi+\delta}^{2 \pi-\delta} d \phi \mathrm{G}_{1}(x, y ; \xi)
$$

the reflection and transmission coefficients tend to zero and decrease like

$$
\begin{gathered}
r(\xi) \underset{|\xi| \rightarrow \infty}{\sim}|\xi|^{2} \exp [-2|\xi \sin (\phi)| d / c], \\
t(\xi) \underset{|\xi| \rightarrow \infty}{\sim}|\xi|^{4} \exp [-4|\xi \sin (\phi)| d / c] .
\end{gathered}
$$

The modulus $|\xi|$ can be chosen large enough to make this second part (70) of the integral arbitrarily small. It has been shown that integral (68) tends to zero when the path $\mathcal{C}$ is a semicircle with infinite radius, and thus expression (65) becomes

$$
\mathrm{G}_{1}(x, y ; z)=\sum_{p} \frac{\mathrm{G}_{1}^{(p)}(x, y)}{z-z_{p}},
$$

which shows that the one-dimensional Green's function can be expanded on the quasinormal modes.

Notice that no justification has been provided to show the convergence of the expansion above on the quasinormal modes. This can be made using classical perfectly matched layers like (66). Indeed, the phase of the poles $z_{p}$ tends to zero when $p$ goes to infinity and it is enough for $\alpha$ to have a positive imaginary part (or a positive argument) to make the imaginary part of product $\alpha z_{p}$ positive. The convergence of the series

$$
\mathrm{G}_{\alpha}(x, y ; z)=\mathrm{G}_{1}(\alpha x, \alpha y ; z)=\sum_{p} \frac{\mathrm{G}_{1}^{(p)}(\alpha x, \alpha y)}{z-z_{p}}
$$

is ensured since the coefficients $\mathrm{G}_{1}^{(p)}(\alpha X, \alpha y)$ have exponential decay for $p$ large enough.

\section{ANALYTIC PROPERTIES IN NON-DISPERSIVE SYSTEMS}

In non-dispersive systems, the dielectric permittivity is independent of the frequency. Here, it is assumed that the dielectric constant is given by expression (10) of permittivity where the frequency has been fixed to the real value $z=\omega_{0}$,

$$
\varepsilon\left(\mathbf{x}, \omega_{0}\right)=\varepsilon_{0}-\int_{\mathbb{R}} d v \frac{\sigma(\mathbf{x}, v)}{\omega_{0}^{2}-v^{2}} .
$$

The Helmholtz operator for the corresponding non-dispersive system is

$$
\left[\mathrm{H}_{d}(z) \mathbf{E}\right](\mathbf{x})=z^{2} \varepsilon\left(\mathbf{x}, \omega_{0}\right) \mu_{0} \mathbf{E}(\mathbf{x})-\partial_{\mathbf{x}} \times \partial_{\mathbf{x}} \times \mathbf{E}(\mathbf{x}) .
$$

In this paper, it is proposed to exploit the new degree of freedom offered by the second complex frequency $\xi$ in $\mathrm{H}(z, \xi)$ [see Sec. II C and Eq. (32)] to analyze the analytic properties 
of the non-dispersive Helmholtz operator $\mathrm{H}_{d}(z)$. The identification of $\mathrm{H}_{d}(z)$ with expression (30) leads to

$$
z\left[\varepsilon\left(\mathbf{X}, \omega_{0}\right)-\varepsilon_{0}\right]=\xi\left[\varepsilon(\mathbf{x}, \xi)-\varepsilon_{0}\right] .
$$

The permittivities are replaced by their Kramers-Kronig expressions (74) and (12), which provides the following condition:

$$
z \int_{\mathbb{R}} d v \frac{\sigma(\mathbf{x}, v)}{\omega_{0}^{2}-v^{2}}=\int_{\mathbb{R}} d v \frac{\sigma(\mathbf{x}, v)}{\xi-v}
$$

A sufficient condition to ensure this equation is

$$
z(\xi-v)=\omega_{0}^{2}-v^{2} \Longleftrightarrow \xi=v+\frac{\omega_{0}^{2}-v^{2}}{z} .
$$

In that case, the imaginary part of $\xi$ is related to the one of $z$ according to

$$
\operatorname{Im}(\xi)=\operatorname{Im}(z) \frac{v^{2}-\omega_{0}^{2}}{|z|^{2}} .
$$

In order to preserve the analytic properties of the inverse Helmholtz operator, it is necessary to have $\operatorname{Im}(\xi) \geq 0$. Since $\operatorname{Im}(z)>0$, it is found that the difference $v^{2}-\omega_{0}^{2}$ has to be positive. This condition is realized if the function $\sigma(\mathbf{x}, v)$ vanishes for frequency $v$ smaller than a frequency $v_{0}>\omega_{0}$. The resulting dielectric constant is given by

$$
\varepsilon\left(\mathbf{X}, \omega_{0}\right)=\varepsilon_{0}+\int_{|v| \geq v_{0}} d v \frac{\sigma(\mathbf{x}, v)}{v^{2}-\omega_{0}^{2}} .
$$

The function under the integral is strictly positive and purely real. Thus the dielectric constant $\varepsilon\left(\mathbf{X}, \omega_{0}\right)$ is real, positive, and takes values greater than $\varepsilon_{0}$ : it describes a transparent dielectric. Notice that this can be established directly from Eq. (75) with $\varepsilon\left(\mathbf{X}, \omega_{0}\right)>0$. However, the present method, leading to the condition $\varepsilon\left(\mathbf{X}, \omega_{0}\right)>\varepsilon_{0}$, is more restrictive since it also contains a causality requirement for the permittivity, as shown hereafter.

It has been established that the analytic properties of the inverse Helmoltz operator can be preserved in non-dispersive systems if the permittivity is the one of transparent dielectric. This analyticity property implies that such non-dispersive systems are physically acceptable. Indeed, it can be checked that the causality principle is preserved for the solution $\boldsymbol{E}(\mathbf{x}, t)$ of Maxwell's equation. Let $\boldsymbol{J}(\mathbf{x}, t)$ be a current source switched on at $t=0$, and hence $\boldsymbol{J}(\mathbf{x}, t)=0$ for $t<0$. Then, after the frequency decomposition, the current source

$$
\mathbf{J}(\mathbf{x}, z)=\int_{0}^{\infty} d t \exp [i z t] \mathbf{J}(\mathbf{x}, t)
$$

is an analytic function in the upper half plane of the complex frequencies $z$. The time dependent electric field is given by

$$
\boldsymbol{E}(\mathbf{x}, t)=\frac{1}{2 \pi} \int_{\Gamma} d z \exp [-i z t] \mathrm{H}_{e}(z)^{-1}\left(i z \mu_{0}\right) \mathbf{J}(\mathbf{x}, z),
$$

where $\Gamma$ is an horizontal line, parallel to the real axis, in the upper half plane. For negative time $t$, the integral can be computed by closing the line $\Gamma$ by a semicircle with infinite radius in the upper half plane. Since all the functions are analytic, it is found that the electric field vanishes for negative times: $\boldsymbol{E}(\mathbf{x}, t)=0$ if $t<0$. Hence, the causality principle is preserved.

In addition, it can be checked that the light velocity $v$ is always smaller than the one in vacuum $c$ since the dielectric constant takes values larger than $\varepsilon_{0}$,

$$
v=\frac{1}{\sqrt{\varepsilon\left(\mathbf{X}, \omega_{0}\right) \mu_{0}}} \leq c=\frac{1}{\sqrt{\varepsilon_{0} \mu_{0}}} .
$$

Notice that if the analyticity property is derived directly from Eq. (75), then the situation $\varepsilon_{0}$ $>\varepsilon\left(\mathbf{x}, \omega_{0}\right)>0$ can occur, which is not physically acceptable: it is in contradiction with the causality requirement for the permittivity and with a light velocity below the one in vacuum $c$. 
Finally, it is stressed that expression (80), obtained for the permittivity and derived from Eq. (76), is merely a sufficient condition which might be too strong. This condition means that a physically acceptable description of a metallic behaviour with $\varepsilon\left(\mathbf{x}, \omega_{0}\right)<0$, ultra-refraction with $\varepsilon\left(\mathbf{X}, \omega_{0}\right)<\varepsilon_{0}$ or absorptive materials, must include frequency dispersion.

\section{CONCLUSION}

Analytic properties of the inverse Helmholtz operator and the electromagnetic Green's function have been established. These properties follow the causality principle and the passivity requirement in frequency dispersive and absorptive media. Notably, the general inverse Helmholtz operator has been shown to be analytic in the domain $\operatorname{Im}(z)-c\left|k^{\prime \prime}\right|>0$ of complex frequencies $z$ and complex wavevectors $\mathbf{k}=\mathbf{k}^{\prime}+i \mathbf{k}^{\prime \prime}$ (Sec. II D). Moreover, it has been shown that an additional degree of freedom (the second frequency $\xi$ ) can be introduced in the inverse Helmholtz operator, which remains analytic as soon as $\operatorname{Im}(\xi) \geq 0$ (Sec. II C). This additional frequency has been then exploited to retrieve that causal systems with non-dispersive permittivity must have purely real dielectric constant taking values above the vacuum permittivity $\varepsilon_{0}$ (Sec. VI). Finally, Kramers-Kronig expressions have been established for the inverse Helmholtz operator and the electromagnetic Green's function (Sec. IV). These Kramers-Kronig expressions can be used as a starting point to extend the well-known eigenmodes expansion of Green's function in the case of a closed cavity. ${ }^{11}$ In particular, it has been shown in the situation of a single dispersive layer that Green's function can be developed on the quasinormal modes, ${ }^{12-14}$ which confirms previous results in the literature. ${ }^{15,16}$

The results established in this paper may be used to calculate time-dependent electromagnetic fields $s^{5,6}$ and to obtain rigorous expansion on quasinormal modes ${ }^{12-16}$ in dispersive and absorptive open systems.

All these results can be extended to magneto-electric materials. ${ }^{19}$ Indeed, in that case, it is enough to add the permeability $\mu(\mathbf{x}, z)$ in the expression of the matrix $\mathrm{V}(\mathbf{x}, z)$,

$$
\mathrm{V}(\mathbf{x}, z)=\left[\begin{array}{lr}
z\left[\varepsilon(\mathbf{x}, z)-\varepsilon_{0}\right] & 0 \\
0 & z\left[\mu(\mathbf{x}, z)-\mu_{0}\right]
\end{array}\right],
$$

and then to apply the arguments proposed in this paper. Also, the analytic properties with respect to the wavevector can be used to extend the Kramers-Kronig expressions (like in Sec. IV) to spatial dispersion.

\section{ACKNOWLEDGMENTS}

Anne-Laure Fehrembach, Sébastien Guenneau, and Gabriel Soriano (Institut Fresnel, Marseille, France) are acknowledged for fruitful discussions. This work was supported by the French National Agency for Research (ANR) under the project "Resonance" (ANR-16-CE24-0013). This work is dedicated to Adriaan Tip.

\section{APPENDIX A: BEHAVIOR OF THE PERMITTIVITY AT $|z| \rightarrow \infty$}

According to estimate (17), let $C(z)$ be defined by

$$
C(z)=z^{2}\left[\varepsilon(\mathbf{x}, z)-\varepsilon_{0}\right]+\left[\partial_{t} \chi\right]\left(\mathbf{x}, 0^{+}\right),
$$

so that (17) becomes equivalent to $C(z) \rightarrow 0$ at the limit $|z| \rightarrow \infty$. The function $C(z)$ is expressed using the Kramers-Kronig relation (10) and the sum rule (16),

$$
C(z)=-\int_{\mathbb{R}} d v \sigma(\mathbf{x}, v) \frac{v^{2}}{z^{2}-v^{2}} .
$$

The integral is split into two parts as follows: $C_{-}(z)$ for $|v|<V$ and $C_{+}(z)$ for $|v|>V$. Let $z$ be written as $z=|z| \exp [i \phi]$, then it is obtained that $\left|z^{2}-v^{2}\right| \geq v^{2} \sin ^{2} \phi$, and the coefficient $C_{+}(z)$,

$$
\left|C_{+}(z)\right| \leq \frac{1}{\sin ^{2} \phi} \int_{|v|>V} d v \sigma(\mathbf{x}, v),
$$


can be made arbitrarily small for $V$ large enough since $\sigma(\mathbf{x}, v)$. Notice that this first step is independent of the modulus of $z$. Next, the modulus of $|z|$ is taken larger than $V$, and the second part of the integral is bounded by

$$
\left|C_{-}(z)\right| \leq \frac{V^{2}}{|z|^{2}-V^{2}} \int_{|v|>V} d v \sigma(\mathbf{x}, v),
$$

which can be made arbitrarily small for $|z|$ large enough. Hence the asymptotic behavior (17) is valid.

\section{APPENDIX B: THE PERMITTIVITY $\varepsilon(\mathbf{x}, z)$ AS AN INTEGRABLE FUNCTION OF THE REAL PART $\omega$ OF $z$}

The integral in (18) is expressed using the Kramers-Kronig relation (10),

$$
\begin{aligned}
\int_{\mathbb{R}} d \omega\left|\varepsilon(\mathbf{x}, z)-\varepsilon_{0}\right| & =\int_{\mathbb{R}} d \omega\left|\int_{\mathbb{R}} d v \frac{\sigma(\mathbf{x}, v)}{z^{2}-v^{2}}\right| \\
& \leq \int_{\mathbb{R}} d \omega \int_{\mathbb{R}} d v \frac{\sigma(\mathbf{x}, v)}{|z-v||z+v|} .
\end{aligned}
$$

Using that $|a|^{2}+|b|^{2} \geq 2|a||b|$, it is found that the last integral is bounded by

$$
\begin{array}{r}
\frac{1}{2} \int_{\mathbb{R}} d \omega \int_{\mathbb{R}} d v \sigma(\mathbf{x}, v)\left[\frac{1}{|z-v|^{2}}+\frac{1}{|z+v|^{2}}\right] \\
=\int_{\mathbb{R}} d v \sigma(\mathbf{x}, v) \int_{\mathbb{R}} d \omega \frac{1}{|z-v|^{2}} .
\end{array}
$$

The last integral over the variable $\omega$ can be calculated by closing the real line $\mathbb{R}$ by a semi-circle,

$$
\int_{\mathbb{R}} d \omega \frac{1}{(\omega-v)^{2}+\eta^{2}}=\frac{\pi}{\eta}
$$

Hence it is obtained that

$$
\int_{\mathbb{R}} d \omega\left|\varepsilon(\mathbf{x}, z)-\varepsilon_{0}\right| \leq \frac{\pi}{\eta} \int_{\mathbb{R}} d v \sigma(\mathbf{x}, v)
$$

which, combined with the sum rule (16), leads to (18).

\section{APPENDIX C: THE ANALYTICITY PROPERTY OF THE INVERSE HELMHOLTZ OPERATOR}

In this section, the analyticity property of the inverse Helmholtz operator is shown using the first resolvent formula. ${ }^{22}$ Denoting $\mathrm{M}(z)=\mathrm{M}_{0}(z)+\mathrm{V}(\mathbf{x}, z)$, the difference of the inverses at $z$ and $z_{0}$ is

$$
\mathrm{M}(z)^{-1}-\mathrm{M}\left(z_{0}\right)^{-1}=\mathrm{M}(z)^{-1}\left(z_{0}-z\right) \mathrm{AM}\left(z_{0}\right)^{-1},
$$

where, using expression (12) for $\mathrm{V}(\mathbf{x}, z)$,

$$
\mathrm{A}=\left[\begin{array}{ll}
1+m & 0 \\
0 & 1
\end{array}\right], \quad m=\varepsilon_{0}^{-1} \int_{\mathbb{R}} d v \frac{\sigma(\mathbf{x}, v)}{(z-v)\left(z_{0}-v\right)} .
$$

The operator $A$ is bounded thanks to condition (16), and $\mathrm{M}\left(z_{0}\right)^{-1}$ is bounded by $1 / \operatorname{Im}\left(z_{0}\right)$. The identity (C1) implies

$$
\begin{aligned}
\mathrm{M}(z)^{-1} & =\mathrm{M}\left(z_{0}\right)^{-1}\left[1-\left(z_{0}-z\right) \operatorname{AM}\left(z_{0}\right)^{-1}\right]^{-1} \\
& =\mathrm{M}\left(z_{0}\right)^{-1}\left\{1+\sum_{p}\left(z_{0}-z\right)^{p}\left[\operatorname{AM}\left(z_{0}\right)^{-1}\right]^{p}\right\}
\end{aligned}
$$

The last series converges in norm provided $\left|z-z_{0}\right|$ is smaller than the inverse of the norm of $\left[\operatorname{AM}\left(z_{0}\right)^{-1}\right]$. This power series expansion shows that the inverse $\left[\mathrm{M}_{0}(z)+\mathrm{V}(\mathbf{x}, z)\right]^{-1}$ is an analytic function if $\operatorname{Im}\left(z_{0}\right)>0$. 


\section{APPENDIX D: KRAMERS-KRONIG RELATIONS FOR THE INVERSE HELMHOLTZ OPERATOR}

The starting point is expression (47) of $\mathrm{R}(z)$ as the inverse Laplace transform of Eq. (45). Using integration by parts, this expression becomes

$$
\mathrm{R}(z)=-\frac{1}{2 \pi} \int_{0}^{\infty} d t \frac{\exp [i z t]}{i z} \partial_{t} \mathrm{X}(t)
$$

since the values at the bounds vanish $[\mathrm{X}(t)$ is continuous and vanishes at $t=0]$. Let $\xi=v+i \zeta$ be a complex number with positive imaginary part such that $\operatorname{Im}(z)=\eta>\zeta>0$. Then, Eq. (D1) implies

$$
i \xi \mathrm{R}(\xi)=-\frac{1}{2 \pi} \int_{0}^{\infty} d t \exp [i \xi t] \partial_{t} \mathrm{X}(t)
$$

and the self-adjoint part is

$$
\begin{aligned}
& i \xi \mathrm{R}(\xi)-i \bar{\xi} \mathrm{R}(\xi)^{\dagger}=-\frac{1}{2 \pi} \int_{0}^{\infty} d t \exp [-\zeta t] 2 \cos [v t] \partial_{t} \mathrm{X}(t) \\
& =-\frac{1}{2 \pi} \int_{\mathbb{R}} d t \exp [i v t] \exp [-\zeta|t|]\left\{\partial_{t} \mathrm{X}(t)+\partial_{t} \mathrm{X}(-t)\right\} .
\end{aligned}
$$

The inverse Fourier transform is used: for $t>0$,

$$
\frac{1}{2 \pi} \partial_{t} \mathrm{X}(t)=\theta(t) \int_{\Gamma_{\zeta}} d \xi \exp [-i \xi t] \frac{\xi \mathrm{R}(\xi)-\bar{\xi} \mathrm{R}(\xi)^{\dagger}}{2 i \pi} .
$$

Injecting this expression in (D1) yields

$$
\mathrm{R}(z)=-\int_{0}^{\infty} d t \frac{\exp [i z t]}{i z} \int_{\Gamma_{\zeta}} d \xi \exp [-i \xi t] \frac{\xi \mathrm{R}(\xi)-\bar{\xi} \mathrm{R}(\xi)^{\dagger}}{2 i \pi}
$$

Next, by analogy with the function $\sigma(\mathbf{x}, v)$ defined by (11), the following quantity is considered:

$$
\mathrm{D}(\xi)=\frac{1}{2 i \pi}[\xi \mathrm{R}(\xi)-\bar{\xi} \mathrm{R}(-\bar{\xi})],
$$

which is self-adjoint [but, contrary to $\sigma(\mathbf{x}, v)$, it is not positive since it is the difference of the one in the media minus the one in vacuum]. The integral over the time in (D5) is performed,

$$
\begin{aligned}
\mathrm{R}(z) & =-\int_{\Gamma_{\zeta}} d \xi \frac{1}{i z} \frac{-1}{i(z-\xi)} \mathrm{D}(\xi) \\
& =-\int_{\Gamma_{\zeta}} d \xi \frac{\mathrm{D}(\xi)}{z^{2}-\xi^{2}} \frac{z+\xi}{z}
\end{aligned}
$$

The integral over $v$ in (D4) and above is independent of the imaginary part $\zeta$ of $\xi$. Hence the integral in (D4) and above can be considered in the limit $\zeta \downarrow 0$. Let $\mathrm{D}(v)$ be defined by

$$
\mathrm{D}(v)=\lim _{\zeta \downarrow 0} \mathrm{D}(v+i \zeta)
$$

and it is left invariant when $v$ is changed in $-v$ [as well as in $\mathrm{D}(\xi)$ ]. It implies the following expressions:

$$
\begin{aligned}
\mathrm{R}(z) & =-\int_{\mathbb{R}} d v \frac{\mathrm{D}(v)}{z^{2}-v^{2}} \quad \operatorname{Im} z>0, \\
z \mathrm{R}(z) & =-\int_{\Gamma_{\zeta}} d \xi \frac{\mathrm{D}(\xi)}{z-\xi} \quad \operatorname{Im} z>\operatorname{Im} \xi \geq 0 .
\end{aligned}
$$

These equations define Kramers-Kronig expressions for the inverse Helmholtz operator. 


\section{APPENDIX E: BEHAVIOUR OF REFLECTION AND TRANSMISSION COEFFICIENTS AT LARGE FREQUENCY}

According to expressions (60), the poles $z_{p}$ of the coefficients $r(z)$ and $t(z)$ are given by

$$
r_{0}(z)^{-1}= \pm \exp \left[2 i z \sqrt{\varepsilon_{1}(z)} d / c\right] .
$$

At large frequency modulus, the following estimates hold:

$$
\varepsilon(z) \approx 1-\frac{\sigma}{z^{2}}, \quad r_{0}(z) \approx \frac{\sigma}{4 z^{2}}, \quad \sigma=\sum_{n=0}^{N} \sigma_{n} .
$$

Assuming that the poles have real part much larger than their imaginary part, the poles are located approximately on the curve

$$
\ln \frac{4 z^{2}}{\sigma} \approx \ln \frac{4[\operatorname{Re}(z)]^{2}}{\sigma} \approx-\frac{2 d}{c} \operatorname{Im}(z)
$$

On this curve, the coefficient $r_{0}(z)$ is almost real and Eq. (E3) implies that the real part of the poles is close to $2 \operatorname{Re}\left(z_{p}\right) d / c=p \pi$ with $p$ integer. Hence the expression of the poles $z_{p}$ for $p$ in $\mathbb{Z}$ is

$$
\operatorname{Re}\left(z_{p}\right) \approx \frac{p \pi c}{2 d}, \quad \operatorname{Im}\left(z_{p}\right)=-\frac{c}{d} \ln \frac{2 \operatorname{Re}\left(z_{p}\right)}{\sqrt{\sigma}} .
$$

The residues of the coefficients $r(z)$ and $t(z)$ can be estimated from (60),

$$
r(z) \underset{z \rightarrow z_{p}}{\approx} \frac{ \pm i c /(4 d)}{\left(z-z_{p}\right)}, \quad t(z) \underset{z \rightarrow z_{p}}{\approx} \frac{\mp i c /(4 d)}{\left(z-z_{p}\right)} .
$$

Outside a strip around the curve (E3) on which the poles are located, the behaviour of $r(z)$ and $t(z)$ can be evaluated on the curves $\mathcal{C}_{\beta}$ with $|\beta| \neq 1$ and defined by

$$
r_{0}\left(z_{\beta}\right)=\beta \exp \left[-2 i z_{\beta} \sqrt{\varepsilon_{1}\left(z_{\beta}\right)} d / c\right],
$$

where

$$
r\left(z_{\beta}\right) \underset{\left|z_{\beta}\right| \rightarrow \infty}{\longrightarrow} \frac{\beta}{1-\beta^{2}}, \quad t\left(z_{\beta}\right) \underset{\left|z_{\beta}\right| \rightarrow \infty}{\longrightarrow} \frac{1}{1-\beta^{2}} .
$$

The case where $1>|\beta| \geq 0$ corresponds to the domain delimited by the pole curve (E3) and the real axis. And the limit $\beta \rightarrow 0$ in (E7) provides the same result than the limit

$$
r(z) \underset{\operatorname{Re}(z) \rightarrow \infty}{\longrightarrow} 0, \quad t(z) \underset{\operatorname{Re}(z) \rightarrow \infty}{\longrightarrow} 1 .
$$

As to the limit $\beta \rightarrow \infty$ in (E7), it provides the same result than the limit $|z| \rightarrow \infty$ when the phase $\phi$ is fixed in $z=|z| e^{i \phi}$,

$$
r(z) \underset{|z| \rightarrow \infty}{\longrightarrow} 0, \quad t(z) \underset{|z| \rightarrow \infty}{\longrightarrow} 0 .
$$

${ }^{1}$ J. D. Jackson, Classical Electrodynamics, 3rd ed. (Wiley, New York, 1998).

${ }^{2}$ M. Reed and B. Simon, Scattering Theory, Methods of Modern Mathematical Physics Vol. 3 (Academic Press, 1979).

${ }^{3}$ L. D. Landau, E. M. Lifshitz, and L. P. Pitaevskiı̌, Electrodynamics of Continuous Media, 2nd ed. (Robert Maxwell, M.C., 1984), ISBN: 0-08-030275-0.

${ }^{4}$ L. Brillouin, Wave Propagation and Group Velocity (Academic Press, Inc., New York, London, 1960).

${ }^{5}$ R. E. Collin, "Frequency dispersion limits resolution in veselago lens," Prog. Electromagn. Res. B 19, 233 (2010).

${ }^{6}$ B. Gralak and D. Maystre, "Negative index materials and time-harmonic electromagnetic field," C. R. Phys. 13, 786 (2012).

${ }^{7}$ P. Grosse and V. Offermann, "Analysis of reflectance data using the Kramers-Kronig relation,” Appl. Phys. A: Solids Surf. 52, 138 (1991).

${ }^{8}$ A. V. Tikhonravov, "Some theoretical aspects of thin-film optics and their applications," Appl. Opt. 32, 5417 (1993).

${ }^{9}$ A. V. Tikhonravov, P. W. Baumeister, and K. V. Popov, "Phase properties of multilayers," Appl. Opt. 36, 4382 (1997).

${ }^{10}$ B. Gralak, M. Lequime, M. Zerrad, and C. Amra, "Phase retrieval of reflection and transmission coefficients from KramersKronig relations," J. Opt. Soc. Am. A 32, 456 (2015).

${ }^{11}$ R. Carminati, A. Cazé, D. Cao, F. Peragut, V. Krachmalnicoff, R. Pierrat, and Y. De Wilde, "Electromagnetic density of states in complex plasmonic systems," Surf. Sci. Rep. 70, 1 (2015).

${ }^{12}$ C. Sauvan, J.-P. Hugonin, I. S. Maksymov, and P. Lalanne, "Theory of the spontaneous optical emission of nanosize photonic and plasmon resonators," Phys. Rev. Lett. 110, 237401 (2013). 
${ }^{13}$ Q. Bai, M. Perrin, C. Sauvan, J.-P. Hugonin, and P. Lalanne, "Efficient and intuitive method for the analysis of light scattering by a resonant nanostructure," Opt. Express 21, 027371 (2013).

${ }^{14}$ B. Vial, A. Nicolet, F. Zolla, and M. Commandré, "Quasimodal expansion of electromagnetic fields in open two-dimensional structures," Phys. Rev. A 89, 023829 (2014).

${ }^{15}$ P. T. Leung, S. Y. Liu, and K. Youn, "Completeness and orthogonality of quasinormal modes in leaky optical cavities," Phys. Rev. A 49, 3057 (1994).

${ }^{16}$ P. T. Leung, S. Y. Liu, and K. Youn, "Completeness and time-independent perturbation of the quasinormal modes of an absorptive and leaky cavity," Phys. Rev. A 49, 3982 (1994).

${ }^{17}$ A. Tip, "Linear absorptive dielectric," Phys. Rev. A 57, 4818-4841 (1998).

18 A. Tip, A. Moroz, and J.-M. Combes, "Band structure of absorptive photonic crystals," J. Phys. A: Math. Gen. 33, 6223 (2000).

${ }^{19}$ B. Gralak and A. Tip, "Macroscopic Maxwell's equations and negative index materials," J. Math. Phys. 51, 052902 (2010).

${ }^{20}$ Y. Liu, S. Guenneau, and B. Gralak, "Causality and passivity properties of effective parameters of electromagnetic multilayered structures," Phys. Rev. B 88, 165104 (2013).

${ }^{21}$ C. Cohen-Tannoudji, B. Diu, and F. Lanoë, Quantum Mechanics (Hermann, 1973), Vol. II.

${ }^{22}$ M. Reed and B. Simon, Functional Analysis, Methods of Modern Mathematical Physics Vol. 1, revised and enlarged ed. (Academic Press, 1980).

${ }^{23}$ M. Reed and B. Simon, Fourier Analysis, Self-Adjointness, Methods of Modern Mathematical Physics Vol. 2 (Academic Press, 1975).

${ }^{24}$ M. Reed and B. Simon, Analysis of Operators, Methods of Modern Mathematical Physics Vol. 4 (Academic Press, 1978).

${ }^{25}$ C.-A. Guérin, B. Gralak, and A. Tip, "Singularity of the dyadic Green's function for heterogeneous dielectrics,” Phys. Rev. E 75, 056601 (2007).

${ }^{26}$ B. Gralak, G. Arismendi, B. Avril, A. Diatta, and S. Guenneau, "Analysis in temporal regime of dispersive invisible structures designed from transformation optics," Phys. Rev. B 93, 121114(R) (2016).

${ }^{27}$ M. I. Abdelrahman and B. Gralak, "Electromagnetic wave propagation through a slab of a dispersive medium," e-print arXiv http://arxiv.org/abs/1610.03639 (submitted).

${ }^{28}$ H. van der Lem, A. Tip, and A. Moroz, "Band structure of absorptive two-dimensional photonic crystals," J. Opt. Soc. Am. B 20, 1334 (2003).

${ }^{29}$ J.-M. Combes, B. Gralak, and A. Tip, "Spectral properties of absorptive photonic crystals," in Waves in Periodic and Random Media, Contemporary Mathematics (American Mathematical Society, 2003), Vol. 339, p. 1.

${ }^{30}$ Y. Brûlé, B. Gralak, and G. Demésy, "Calculation and analysis of the complex band structure of dispersive and dissipative two-dimensional photonic crystals," J. Opt. Soc. Am. B 33, 691 (2016).

${ }^{31}$ A. Raman and S. Fan, "Photonic band structure of dispersive metamaterials formulated as a hermitian eigenvalue problem," Phys. Rev. Lett. 104, 087401 (2010).

32 J.-P. Berenger, "A perfectly matched layer for the absorption of electromagnetic waves,” J. Comput. Phys. 114, 185 (1994) 